\title{
BMJ Open Depression and anxiety symptoms to COVID-19 outbreak among the public, medical staff and patients during the initial phase of the pandemic: an online questionnaire survey by a WeChat Mini Program
}

Xianglan Wang, ${ }^{1}$ Jiong Tao, ${ }^{1}$ Qi Zhu, ${ }^{1}$ Xiuhua Wu, ${ }^{1}$ Tong Li, ${ }^{1}$ Chongbang Zhao, ${ }^{1}$ Weirui Yang, ${ }^{1}$ Xiaoying Wang, ${ }^{2}$ Jinbei Zhang, ${ }^{1}$ Nianhong Guan (i) ${ }^{1}$

To cite: Wang X, Tao J, Zhu Q, et al. Depression and anxiety symptoms to COVID-19 outbreak among the public, medical staff and patients during the initial phase of the pandemic: an online questionnaire survey by a WeChat Mini Program. BMJ Open 2021;11:e046350. doi:10.1136/ bmjopen-2020-046350

- Prepublication history for this paper is available online. To view these files, please visit the journal online (http://dx.doi. org/10.1136/bmjopen-2020046350).

$\mathrm{XLW}$ and JT contributed equally. $\mathrm{XYW}$ and $\mathrm{NG}$ contributed equally.

Received 28 October 2020 Accepted 10 May 2021

Check for updates

(c) Author(s) (or their employer(s)) 2021. Re-use permitted under CC BY-NC. No commercial re-use. See rights and permissions. Published by BMJ.

${ }^{1}$ Department of Psychiatry, Third Affiliated Hospital of Sun Yat-sen University, Guangzhou, China ${ }^{2}$ Department of Artificial Intelligence, Third Affiliated Hospital of Sun Yat-sen University, Guangzhou, China

Correspondence to Dr Nianhong Guan; kuwanhong@163.com and Dr Xiaoying Wang; 18680501034@163.com

\section{ABSTRACT}

Objectives To survey anxiety and depression symptoms to COVID-19 outbreak in the public, medical staff and patients during the initial phase of the pandemic.

Design Cross-sectional online survey administered through WeChat Mini Program using Chinese versions of Zung Self-rating Depression Scale and Zung Self-rating Anxiety Scale.

Setting Guangzhou, China.

Participants 47378 public, 1512 medical staff and 125 patients with COVID-19.

Results Higher rates of depression (47.8\%) and anxiety symptoms (48.7\%) were shown by patients who were screened positive compared with those of the public $(35.6 \%, 25.7 \%)$ or medical staff $(15.4 \%, 13.3 \%)$. The professional identity of a nurse, conditions of 'with an infected family member' and 'working at the frontline' were risk factors to depression or anxiety symptoms for the medical staff. Younger age, lower educational level, female and not having adequate masks were the risk factors for the public.

Conclusion The COVID-19 outbreak increased people's depression or anxiety emotion responses, which varied extensively among the patients, public and medical staff.

\section{INTRODUCTION}

Although 1 year has passed since the COVID-19 was first diagnosed and reported in the Hubei province of China, it is still pathologically and psychologically affecting people worldwide. ${ }^{1}$ SARS-CoV-2 impairs the central nervous system to result in mental disturbances in few patients with COVID-19 by the direct infection or subsequent influences from abnormal immune system functions or severe somatic diseases. ${ }^{2} 3$ The COVID-19 mainly impacted people's mental health as a psychological stressor arouses
Strengths and limitations of this study

- The standardised common scales of Zung Self-rating Depression Scale and Zung Self-rating Anxiety Scale were used to measure the emotional responses.

- A very large sample of the public during the initial phase of the COVID-19 pandemic was enrolled.

- Different emotional responses and affecting factors were analysed among the public, health workers and patients with COVID-19.

- The actual identities of participants were not acquired because of online surveying.

from its infectious and fatal natural features and special control and preventive social measures. ${ }^{45}$

According to the previous studies, anxiety and depression symptoms were most frequent among the patients, public and medical staff during the initial stage of the COVID-19 outbreak. ${ }^{6-12}$ The stress levels vary among the different populations during the pandemic due to different threat intensities. The patients with COVID-19 and frontline medical staff were considered at higher risk for mental disorders than the public. However, only a few studies compared psychological responses to the COVID-19 pandemic among these populations.

This online psychological self-rating survey had been conducted among the public, medical staff and patients from 31 January to 14 February 2020. At that time, the most positive and restrictive measures that were being carried out nationally to prevent the epidemic and the remarkable biological feature of infection of COVID-19 made the 
online survey the more optimal way to collect evidence for psychological intervention to the outbreak.

\section{METHODS}

\section{Participants}

The present online psychological self-rating survey was released at the WeChat official account of the Third Affiliated Hospital of Sun Yat-sen University and readily available to people nationwide who want to do it. The inclusion criteria are as follows: (1) informed consent; (2) male or female; (3) 12 years of age or older; (4) education at or above primary school level. Exclusion criteria include: (1) lack of essential information such as age and gender; (2) incompletion of psychological scales; (3) selection of more than one edition of the survey. To ensure that they could read and understand the text of the questionnaire or psychological scales and complete it independently, we required participants to have an educational level of the primary school or above and more than 12 years old.

\section{Instruments}

A study team comprising psychiatrists and artificial intelligence (AI) engineers from the Third Affiliated Hospital of Sun Yat-sen University designed the psychological selfrating survey program. Three editions and three subsequent access paths were prepared: the public, medical staff and patient edition.

All the three editions comprised five parts, including an informed consent form and instructions for the survey, a questionnaire for demographic data and questions regarding the epidemic, the Chinese version of the Zung Self-rating Anxiety Scale (ZSAS), the Chinese version of the Zung Self-rating Depression Scale (ZSDS), and a survey outcome and relevant self-adjustment recommendations or methods for getting professional help. In the first part of the informed consent, the minor was instructed to get agreement from parents or guidance before he started this survey.

The demographic information involved gender, age, marriage, occupation and location. Also, certain specific survey questions were included in various editions. For instance, related questions in the survey for the public included: 'Do you have enough masks?' and 'Have your family members been infected?'. The survey for medical staff comprised questions such as: 'Have you worked at the isolation ward or fever clinic?', 'Have your family members been infected?' and 'Have you been kept on-call to leave for Hubei province to assist treatment of COVID-19?'.

The Chinese versions of $\mathrm{ZSDS}^{13}$ and $\mathrm{ZSAS}^{14}$ were two common standardised scales with good validity and reliability used for assessing the depression and anxiety severities, respectively, during the past 1 week. The scales of ZSDS and ZSAS were commonly used to evaluate depression and anxiety symptoms in clinical work and research since they were introduced to China in the 1980s of the 20th century. Many previous studies had used these two scales to evaluate the depression or anxiety symptoms in Chinese population including adolescents. ${ }^{15-18}$ Among patients with depression, the correlation coefficient between scores of ZSDS and scores of Hamilton Depression Scale was $0.84 .{ }^{13}$ Among patients with neurosis, the correlation coefficient between scores of ZSAS and scores of Hamilton Anxiety Scale was $0.365 .{ }^{14}$ These two scales had the same number of items (20) and similar score calculation methods. The scores were obtained as follows: the crude scale score (CS) was equal to the sum of each item score, and the scale standardised score (SS) or index score was equal to CS multiplied by 1.25. Cut-off scores of the severity of anxiety as per ZSAS SS were set as none: if $<50$; mild: 50-59; moderate: $60-69$; and severe: $>70$. A cut-off score of screening positive for anxiety symptoms was 50 of the ZSAS SS. Cut-off scores of the severity of depression per ZSDS SS were set as none: if $<53$; mild: 53-62; moderate: 63-72; and severe: $>72$. A cut-off score of screening positive for depression symptoms was 53 of the ZSDS SS. ${ }^{19}$

\section{Procedure}

This self-rating system was designed in the form of a WeChat Mini Program that people could use through the smart mobile phone to read the QR code and authorise a WeChat application to add this mini program, and then select one of the three optimal editions for self-assessment.

\section{Data cleaning}

First, all test data using an age marker with the help of one input by the AI engineers were cleaned. Second, except for the last complete data, the rest of the data from the same person in a single day were discarded. Except for the data of the first day, all the duplicates of the data entered by the same person on other days were discarded. Third, data without both the ZSDS and ZSAS scores were deleted. Fourth, data with logical inconsistencies such as the contradiction between choosing the medical staff edition and age of $<18$ years, among occupation and educational levels and age, selecting all three editions to respond, etc (figure 1).

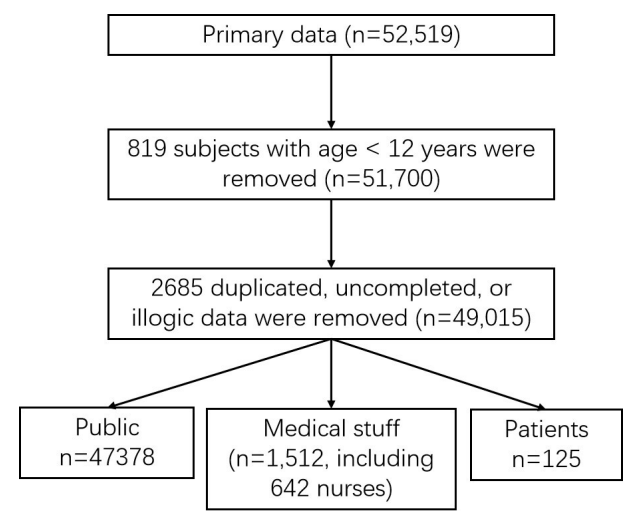

Figure 1 The flow chart of data cleaning. 


\section{Statistical analysis}

To build the data set and conduct statistical analysis, the IBM SPSS Statistics for Windows V.26.0 (IBM) was used. Comparisons of group ZSDS SS or ZSAS SS were performed by t-test or one-way analysis of variance (ANOVA). Distributions of varying ratios of severity levels were compared between groups through the $\chi^{2}$ test. The binary variable of screening positive for depression or anxiety symptoms, 0 -negative and 1 -positive, was the dependent variable. Demographic factors such as gender, age, education, occupation, location, whether any family member is infected, whether working at the isolation ward and whether working at fever clinics were the independent variables. Logistic regression was performed to analyse the relevant factors of depression or anxiety responses to the epidemic. As the natural limitation of the online survey, there were several missing values in the data set. We used a set of measures to handle missing values as follows: first, during the data cleaning, according to the inclusion and exclusion criteria to screening the data of every participant; second, in the processes of analyses of t-test and ANOVA, we chose the option of 'exclude cases analysis by analysis' to avoid the influences of missing values on statistical results; third, the $\chi^{2}$ test and binary logistic regression were abound to automatically exclude missing values of involved variables during data processing. We did not use other specific statistical function to handle missing values. The statistical level of significance was set at alpha $=0.05$.

\section{Patient and public involvement}

No patient or the public were involved in the design, conduct, reporting or dissemination plans of the research.

\section{RESULTS}

\section{Overall view and demographic data}

Data pertaining to 52519 cases gathered from 31 January to 14 February 2020 were exported from the program computer server. The respondents geographically belonged to 34 provinces of China, of which Guangdong (28 068, 53.4\%), Henan (1921, 3.7\%), Hubei (1643, $3.1 \%)$, Hunan (1407, 2.7\%), Beijing (1356, 2.6\%), Shanghai (1050, 2.0\%), Sichuan (986, 1.9\%), Guangxi (932, 1.8\%), Shandong (916, 1.7\%) and Jiangsu (893, $1.7 \%$ ) were the top 10 provinces. Following data cleaning, data of 49015 cases were pooled for further analysis, comprising 47378 public, 1512 medical staff and 125 patients. Valid data included 29867 cases of ZSDS and 43149 cases of ZSAS. Among 1512 cases of medical staff, 642 reported their occupations as a nurse.

Overall, 32801 people had provided the information on their gender (among the public, 5098 were males $(35.8 \%)$ and 9126 were females $(64.2 \%)$; among medical staff, 294 were males $(19.7 \%)$ and 1202 were females $(80.3 \%)$; among patients, 35 were males $(34.0 \%)$ and 68 were females $(66.0 \%))$.
A total of 15561 people had provided information regarding their age. The average age of the 102 cases belonging to the patient group was $32 \pm 11$ years (range: 14-70 years). The average age of the 13980 cases belonging to the public group was $32 \pm 9$ years (range: $13-88$ years), and the group was divided into five subgroups according to age: $<25$ years $(21.5 \%)$; $25-34$ years $(44.3 \%)$; $35-44$ years $(24.3 \%) ; 45-55$ years $(7.8 \%)$; and $>55$ years $(2.2 \%)$. The average age of 1479 cases belonging to the medical staff group was 33 \pm 8 years (range: 20-62 years), and the group was divided into four subgroups: $20-29$ years (35.6\%); $30-39$ years $(43.7 \%)$; $40-49$ years $(14.7 \%)$; and $>50$ years $(3.8 \%)$.

\section{Differences among the three groups}

The patient group exhibited a screening positive rate of $48.7 \%$ for anxiety symptoms, which was remarkably greater than those of both the public $(25.7 \%)$ and medical staff $(13.3 \%)$ groups $(\mathrm{p}<0.001)$. The patient group also showed a higher screening positive rate of $47.8 \%$ for depression symptoms higher than that of both the public $(35.6 \%)$ and medical staff $(15.4 \%) \quad(\mathrm{p}<0.001)$. For the severity of anxiety, the patient group revealed the highest sum ratios $(25.2 \%)$ of both moderate and severe among the three groups, and those of the public and medical staff groups were $8.7 \%$ and $3.8 \%$, respectively $(p<0.001)$. The sum ratios of moderate and severe depression among the patient, public and medical staff groups were $27.8 \%$, $16.3 \%$ and $4.0 \%$, respectively $(\mathrm{p}<0.001)$. Outcomes of one-way ANOVA of SS and all factor scores of ZSDS and ZSAS suggested that significant differences existed among the three groups. All the scores of the patient group were highest among the three groups, followed by the public group and then the medical staff group (table 1).

\section{Associated factors of ZSDS and ZSAS of the medical staff group \\ Group differences of means and screening positive rates of ZSAS and ZSDS}

One-way ANOVA or t-test outcomes for group differences of ZSAS SS and ZSDS SS means indicated that factors such as age, gender, educational levels, marriage status, medical divisions, doctor or nurse, whether working at isolation wards, whether working at fever clinics and having any family member infected may influence the ZSDS SS or ZSAS SS and screening positive rates for depression and anxiety. People with an infected family member exhibited significantly higher screening positive rates for depression $(43.3 \%)$ and anxiety $(40.5 \%)$ than individuals having none in the family members infected (table 2).

Logistic regression of screening negative or positive for depression and anxiety symptoms among medical staff

Result of screening positive for depression or anxiety was considered as the dependent variable: negative was set as 0 and positive as 1 . The independent variables included age groups, gender, educational levels, 


\begin{tabular}{|c|c|c|c|c|c|}
\hline & Medical staff & Public & Patients & Statistics & $P$ value \\
\hline ZSAS & $n=1502$ & $\mathrm{n}=41534$ & $\mathrm{n}=113$ & & \\
\hline Standard scores* & $37.93 \pm 9.53$ & $42.53 \pm 11.27$ & $48.91 \pm 13.09$ & $\mathrm{~F}=141.201$ & $<0.001$ \\
\hline Screening positive, n (\%) & $200(13.3)$ & $10656(25.7)$ & $55(48.7)$ & $\chi^{2}=149.643$ & $<0.001$ \\
\hline Severity, n (\%) & & & & $\chi^{2}=170.207$ & $<0.001$ \\
\hline None & $1302(86.7)$ & $30878(74.3)$ & $58(52.2)$ & & \\
\hline Mild & $143(9.5)$ & $7037(16.9)$ & $26(22.6)$ & & \\
\hline Moderate & $50(3.3)$ & $2685(6.5)$ & $22(19.1)$ & & \\
\hline Severe & $7(0.5)$ & $934(2.2)$ & $7(6.1)$ & & \\
\hline ZSDS & $n=1269$ & $\mathrm{n}=28508$ & $\mathrm{n}=90$ & & \\
\hline Standard scores* & $39.22 \pm 11.84$ & $47.69 \pm 14.39$ & $53.28 \pm 15.84$ & $F=220.965$ & $<0.001$ \\
\hline Screening positive, $\mathrm{n}(\%)$ & $195(15.4)$ & $10136(35.6)$ & $43(47.8)$ & $\chi^{2}=225.206$ & $<0.001$ \\
\hline Severity, n (\%) & & & & $\chi^{2}=245.460$ & $<0.001$ \\
\hline None & $1074(84.6)$ & $18372(64.4)$ & $47(52.2)$ & & \\
\hline Mild & $145(11.4)$ & $5475(19.2)$ & $18(20.0)$ & & \\
\hline Moderate & $40(3.2)$ & 3171 (11.1) & 15 (16.7) & & \\
\hline Severe & $10(0.8)$ & $1490(5.2)$ & $10(11.1)$ & & \\
\hline
\end{tabular}

*Post hoc Student-Newman-Keuls (S-N-K) test showed statistically significant group differences among all the three groups. ZSAS, Zung Self-rating Anxiety Scale; ; ZSDS, Zung Self-rating Depression Scale.

marriage, medical divisions, whether a doctor or nurse, whether working at isolation wards, whether working at fever clinics and having family member infected. Variables of nurse $(\mathrm{OR}=1.628, \mathrm{p}=0.014)$, with an infected family member $(\mathrm{OR}=3.186, \mathrm{p}=0.006)$ and whether working at isolation wards $(\mathrm{OR}=2.197, \mathrm{p}=0.004)$ entered the regression model of the screening positive for depression (the model $\chi^{2}=23.037, \mathrm{df}=3, \mathrm{p}<0.0001$, Cox and Snell $\mathrm{R}^{2}=0.025$, overall classification percentage $=85.1 \%)$. Variables of nurse $(\mathrm{OR}=1.560, \mathrm{p}=0.025)$, with an infected family member $(\mathrm{OR}=4.041, \mathrm{p}<0.0001)$ and whether working at fever clinics $(\mathrm{OR}=2.194, \mathrm{p}=0.005)$ entered the regression model of the screening positive for anxiety (the model $\chi^{2}=20.892, \mathrm{df}=3$, $\mathrm{p}<0.0001$, Cox and Snell $\mathrm{R}^{2}=0.019$, overall classification percentage $=87.6 \%)($ refer to table 3$)$.

\section{Associated factors of ZSDS and ZSAS of the public group}

Group differences of means and screening positive rates of ZSAS and ZSDS among the public group

One-way ANOVA or t-test outcomes for group differences of ZSAS SS and ZSDS SS means suggested that age, gender, educational levels, marriage status and having enough masks for 1 week may affect the ZSDS SS or ZSAS SS and screening positive rates for depression and anxiety. People with primary education exhibited the highest screening positive rate for depression $(56.3 \%)$ and anxiety symptoms $(30.8 \%)$ than those with other educational levels. People aged $<24$ and 25-34 years suffered higher rates of depression $(37.0 \%, 33.4 \%)$ and anxiety $(28.6 \%, 25.2 \%)$ than individuals of other age groups. Factors such as gender and having enough masks as well as locations also influenced the SS means of ZSDS or ZSAS and screening positive rates for depression or anxiety (table 4 ).
Logistic regression for screening negative or positive for depression and anxiety symptoms in the public

As per the results of stepwise binary logistic regression analysis, variables of age (group of $>55$ years old as the reference category), educational level (group of postgraduates as the reference category), having contracted with confirmed cases, gender (male as the reference), having not enough masks and marriage (single group as the reference category) enter the regression model $\left(\chi^{2}=321.659, \mathrm{df}=14\right.$, $\mathrm{p}<0.0001$; Cox and Snell $\mathrm{R}^{2}=0.028$; overall classification percentage $=77.2 \%)$.

Variables of educational level (group of postgraduates as the reference category), age (group of $>55$ years old as the reference category), gender (male as the reference), having not enough masks, marriage (single group as the reference category) and location distance from Hubei entered the regression model for screening positive for depression symptoms $\left(\chi^{2}=396.076, \mathrm{df}=16, \mathrm{p}<0.0001\right.$; Cox and Snell $\mathrm{R}^{2}=0.044$; overall classification percentage $=70.1 \%$ ). Refer to table 5 for details.

\section{DISCUSSION}

This study throws light on the acute psychological responses of anxiety or depression symptoms of the public, medical staff and patient groups under the COVID-19 pandemic by a nationwide online selfassessment in China. Patients showed significant anxiety or depression symptoms than the other two groups of the public and medical staff, and the respondents of the medical staff group maintained the best mental health status. With regard to the public group, age, educational 
Table 2 Relevant factors of ZSAS and ZSDS of the medical staff

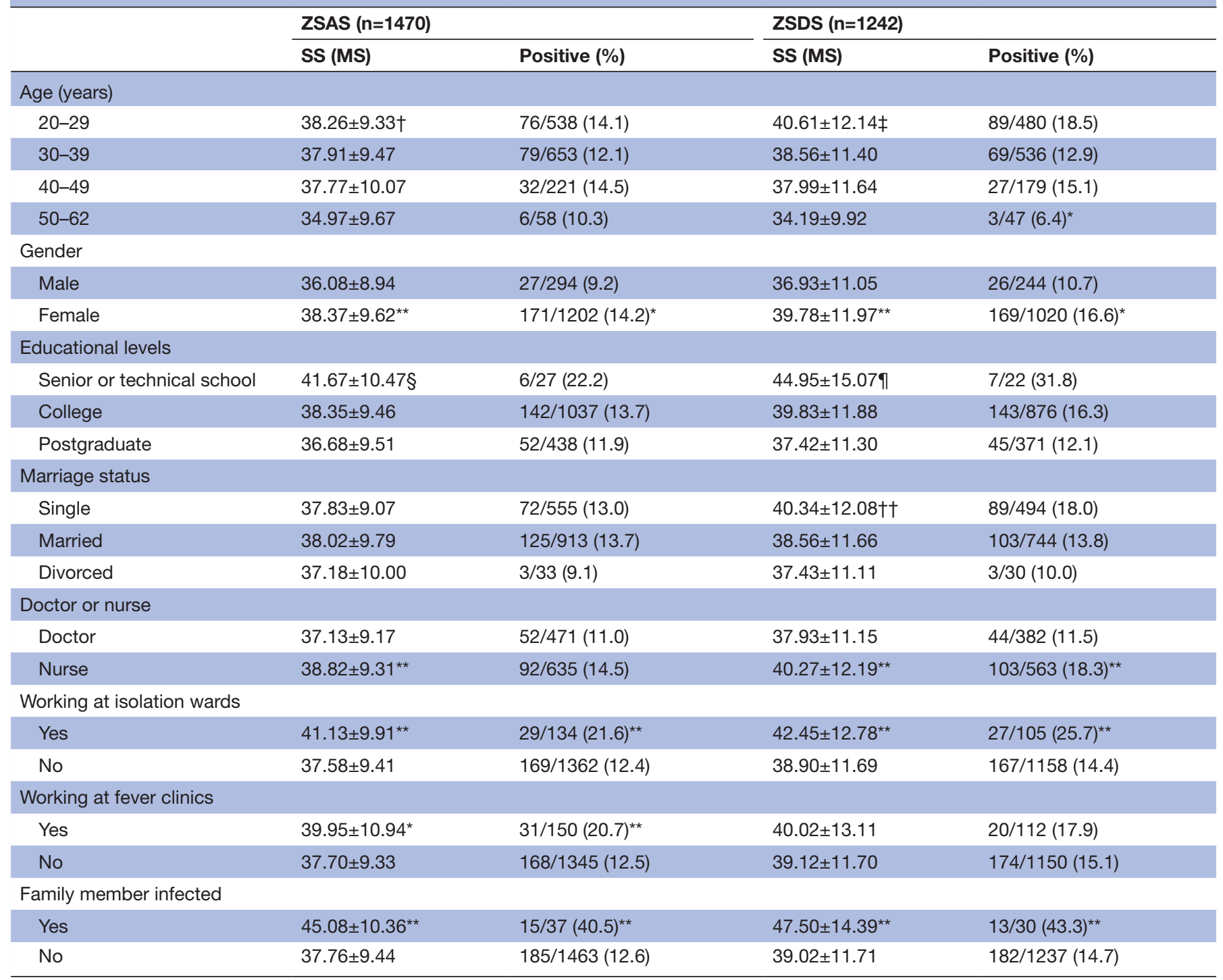

${ }^{*} \mathrm{P}<0.05 ;{ }^{* *} \mathrm{p}<0.01$

tOne-way analysis of variance (ANOVA) F=2.104, $\mathrm{p}=0.098$, post hoc $\mathrm{S}-\mathrm{N}-\mathrm{K}$ : two subsets for alpha $=0.05$ : $>50$ and all other age groups. fOne-way ANOVA F=6.352, $\mathrm{p}<0.0001$, post hoc $\mathrm{S}-\mathrm{N}-\mathrm{K}$ : two subsets for alpha=0.05: group of $>50$ and all other age groups.

$\S$ One-way ANOVA F=6.911, $p=0.001$, post hoc $\mathrm{S}-\mathrm{N}-\mathrm{K}$ : two subsets for alpha $=0.05$ : groups of college and postgraduate, and group of senior or technical school.

१One-way ANOVA F=8.151, $p<0.0001$, post hoc $\mathrm{S}-\mathrm{N}-\mathrm{K}$ : two subsets for alpha=0.05: groups of college and postgraduate, and group of senior or technical school.

††One-way ANOVA $F=3.745, p=0.024$, multiple comparisons of least significant difference (LSD): group differences of mean ZSDS SS between single and married were significant, $\mathrm{p}=0.009$.

MS, Mean \pm SD; SS, scale standardised score; ZSAS, Zung Self-rating Anxiety Scale; ZSDS, Zung Self-rating Depression Scale.

levels, whether having enough masks and marital status were relevant factors for the occurrence of anxiety or depression symptoms. Simultaneously, the medical staff with an infected family member, worked at an isolation ward or fever clinic or serviced as a nurse faced a high risk of depression or anxiety symptoms.

Generally, patients with COVID-19 were considered at the highest risk of psychiatric illness, especially during the acute phase, comorbid anxiety and depression symptoms as the most frequent. Zhang $e t a l^{20}$ reported the results of the psychiatric contact consultation to 105 patients with COVID-19 in isolated wards and showed that the prevalence of anxiety and depression in 105 patients was $61.9 \%$ and $25.7 \%$, respectively. Rogers et at conducted a systematic review and meta-analysis about the psychiatric and neuropsychiatric presentations of severe acute respiratory syndrome (SARS), Middle East respiratory syndrome and COVID-19. The results showed depressed $\operatorname{mood}(32.6 \%)$, anxiety $(35.7 \%)$, insomnia and impaired memory were the most common psychiatric symptoms during the acute stage except the organic confusion symptoms. In agreement with the earlier studies, this study found that patients with COVID-19 were at high risk of poor mental health, and they exhibited a screening 
Table 3 Relevant factors of screening positive for depression or anxiety symptoms among medical staff by stepwise logistic regression analysis

\begin{tabular}{|c|c|c|c|c|c|c|c|c|}
\hline & \multirow[b]{2}{*}{ B } & \multirow[b]{2}{*}{ SE } & \multirow[b]{2}{*}{ Wald } & \multirow[b]{2}{*}{ df } & \multirow[b]{2}{*}{ Significance } & \multirow[b]{2}{*}{$\operatorname{Exp}(B)$} & \multicolumn{2}{|c|}{$95 \% \mathrm{Cl}$ for $\operatorname{Exp}(\mathrm{B})$} \\
\hline & & & & & & & Lower & Upper \\
\hline \multicolumn{9}{|c|}{ Screening positive for depression } \\
\hline Family member infected & 1.159 & 0.420 & 7.603 & 1 & 0.006 & 3.186 & 1.398 & 7.260 \\
\hline Working at isolation ward & 0.787 & 0.272 & 8.402 & 1 & 0.004 & 2.197 & 1.290 & 3.742 \\
\hline \multicolumn{9}{|l|}{ Screening positive for anxiety } \\
\hline Family member infected & 1.396 & 0.388 & 12.936 & 1 & $<0.001$ & 4.041 & 1.888 & 8.649 \\
\hline Working at fever clinics & 0.786 & 0.279 & 7.912 & 1 & 0.005 & 2.194 & 1.269 & 3.792 \\
\hline
\end{tabular}

positive rate of the anxiety of $48.7 \%$ and depression of $47.8 \%$. The unpredictable development trend of COVID-19 brings more stressful and helpless experiences to patients, and they might have to confront more severe impacts on the mind during the acute and long-term phases. A cohort study of 90 patients with SARS conducted by Mak $e t a l^{21}$ evidenced $25.6 \%$ of patients diagnosed with post-traumatic stress disorder, $15.5 \%$ as depressive disorders and $16.5 \%$ as anxiety disorders at 30 months postSARS. Therefore, patients with COVID-19 are likely the

Table 4 Relevant factors of ZSAS and ZSDS of the public group

\begin{tabular}{|c|c|c|c|c|}
\hline & \multicolumn{2}{|l|}{ ZSAS } & \multicolumn{2}{|l|}{ ZSDS } \\
\hline & SS (MS ) & Positive (\%) & SS (MS ) & Positive (\%) \\
\hline \multicolumn{5}{|l|}{ Age (years) } \\
\hline $25-34$ & $42.65 \pm 10.43$ & 1520/6039 (25.2) & $46.92 \pm 13.48$ & $1316 / 3945$ (33.4) \\
\hline $35-44$ & $40.11 \pm 10.12$ & 607/3342 (18.2) & $42.74 \pm 12.63$ & 461/2024 (22.8) \\
\hline \multicolumn{5}{|l|}{ Gender } \\
\hline Male & $40.76 \pm 10.47$ & $1019 / 5098$ (20.0) & $43.84 \pm 13.44$ & $838 / 3221(26.0)$ \\
\hline Female & $42.37 \pm 10.58^{\star *}$ & $2278 / 9126(24.9)^{\star \star}$ & $46.97 \pm 13.63^{\star *}$ & $2049 / 6207(33.0)^{\star \star}$ \\
\hline \multicolumn{5}{|l|}{ Educational level } \\
\hline College & $41.63 \pm 10.48$ & 2219/9726 (22.8) & $45.56 \pm 13.40$ & 1906/6459 (29.5) \\
\hline Postgraduate & $40.71 \pm 10.28$ & $326 / 1563(20.9)$ & $44.27 \pm 13.00$ & $258 / 990(26.1)$ \\
\hline \multicolumn{5}{|l|}{ Marriage } \\
\hline Single & $43.20 \pm 10.88 \dagger \dagger$ & $1734 / 6217(27.9)^{\star \star}$ & $48.27 \pm 14.11 \neq \ddagger$ & $1678 / 4559(36.8)^{\star \star}$ \\
\hline Married & $40.69 \pm 10.05$ & 1444/7474 (19.3) & $43.55 \pm 12.71$ & $1104 / 4535$ (24.3) \\
\hline Divorced & $41.56 \pm 11.27$ & $110 / 488(22.5)$ & $46.16 \pm 13.45$ & $96 / 299(32.1)$ \\
\hline Widowed & $38.60 \pm 11.89$ & 9/58 (15.5) & $43.13 \pm 15.32$ & $9 / 40(22.5)$ \\
\hline \multicolumn{5}{|c|}{ Having enough masks } \\
\hline Yes & $41.05 \pm 10.31$ & 1699/8069 (21.1) & $44.83 \pm 13.42$ & $1456 / 5286(27.5)$ \\
\hline No & $42.72 \pm 10.73^{\star \star}$ & $1611 / 6287(25.6)^{\star \star}$ & $47.16 \pm 13.75^{\star \star}$ & $1427 / 4188(34.1)^{\star \star}$ \\
\hline
\end{tabular}

${ }^{*} \mathrm{P}<0.05 ;{ }^{* *} \mathrm{p}<0.01$

†One-way analysis of variance (ANOVA) F=85.793, $\mathrm{p}<0.0001$; S-N-K: four subsets for alpha=0.05: $>55,45-54,35-44$, and $25-34$ and $<24$.

fOne-way ANOVA F=88.700, $\mathrm{p}<0.0001$; S-N-K: five subsets for alpha $=0.05:>55,45-54,35-44,25-34$ and $<24$.

§One-way ANOVA F=16.662, $\mathrm{p}<0.0001$; S-N-K: four subsets for alpha=0.05: postgraduate and college, senior/technical school, junior school and primary school.

ๆOne-way ANOVA F=21.201, $p<0.0001 ; \mathrm{S}-\mathrm{N}-\mathrm{K}$ : four subsets for alpha=0.05: postgraduate and college, senior/technical school, junior school and primary school.

††One-way ANOVA F=67.220, $\mathrm{p}<0.0001, \mathrm{~S}-\mathrm{N}-\mathrm{K}$ : three subsets for alpha $=0.05$ : widowed, married and divorced, and single.

ł¥One-way ANOVA F=94.184, $\mathrm{p}<0.0001$, S-N-K: two subsets for alpha=0.05: widowed and married and divorced, and single.

MS, Mean \pm SD; SS, scale standardised score; ZSAS, Zung Self-rating Anxiety Scale; ZSDS, Zung Self-rating Depression Scale. 
Table 5 Relevant factors of screening positive for depression or anxiety symptoms in public by stepwise logistic regression analysis

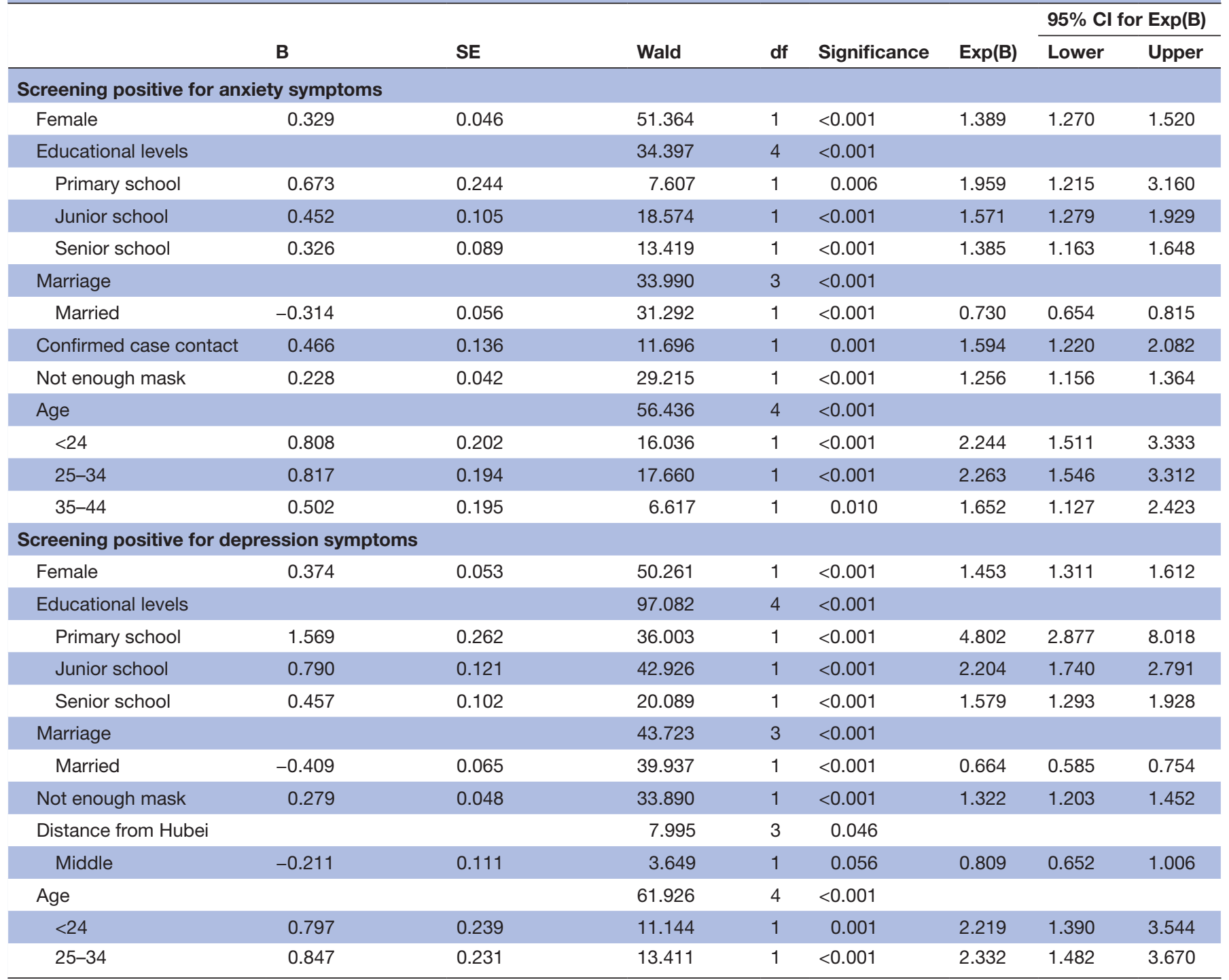

high-risk population and in need of psychological intervention. The early diagnosis and timely intervention may aid in reducing future psychiatric morbidity. In China, as a part of the treatment and prevention policy, a total of 450 psychiatrists or psychologists provided mental assessment, psychiatric contact consultation and psychological intervention by the bedside in isolated wards ${ }^{20}$ in Hubei province according to the guideline of the national health authority. Huang et $a l^{22}$ reported the prevalence of anxiety or depression of 1733 patients with COVID-19 discharged from hospital for 6 months was 23\%. Combined with the present study's findings, the incidence of emotional disturbances in patients would decrease with the recovery, which may be related to the pathological mechanism of the disease, environmental changes and psychological interventions and other factors.

The extensive spread of this infectious and fatal disease may inevitably affect the public's mental health, especially during the initial phase due to huge uncertainty caused by the lack of knowledge regarding it. The systematic review and meta-analysis study by Salari $e t$ at ${ }^{6}$ showed the prevalence of anxiety and depression was $31.9 \%$ and $33.7 \%$ in the general population during the COVID-19 pandemic, respectively. These findings supported the present study results in which the screening positive rate of anxiety and depression in the public was $25.7 \%$ and $35.6 \%$, respectively. As to the relevant factors, in agreement with the earlier studies, ${ }^{83-25}$ this study also found female gender, younger age and lower educational levels are risk factors for people to be anxious and depressed. It was also found that whether having enough precautional material such as masks does not affect the mental status of the public. All these outcomes suggest that the high-risk population needs to be paid more attention and needs psychological intervention for COVID-19 in the future. Supporting with adequate precautional material may be the basis of the intervention. Dealing with the maladaptation to the living pattern adjustments and reconstructing the distorted 
cognitions regarding the epidemic or responses of mind and body should be the key tasks of the psychological intervention. ${ }^{26}{ }^{27}$ In China, many professional institutes of mental health in every city have set up several hot lines through telephone or mobile internet. All these services are entirely free for the public as these are critical to people in severe psychological crisis. ${ }^{28}$ Additionally, as to the children and adolescents, they could have some particular emotional and behavioural reactions due to various stressful factors under the background of the COVID-19 pandemic. This study directly or indirectly reflected their mental health level by evaluating depression or anxiety symptoms. They should be paid more comprehensive attention in psychological assessments and interventions.

Undoubtedly, in this serious public health issue, the medical staff played a key role. While they worked hard to treat and care for the patients, they made great efforts to prevent themselves from infection of COVID-19. It is understood that the medical staff, especially those working at the front line against the virus, were in a highly stressful state. However, they were observed to exhibit the lowest screening positive rate of $13.3 \%$ for anxiety or $15.4 \%$ for depression symptoms than the patients and the public. This finding was similar to a multicentre study in medical staff which reported the overall prevalence of anxiety and depression of $13.9 \%$ and $16.1 \%$ among 274 responders. ${ }^{29}$ The main factors contributing to the low positive rates might be as follows: (1) The time this study investigated was very early in the outbreak. Except in Wuhan and Hubei provinces, medical staff in other districts hardly were exposed to confirmed patients. So, overall, the level of psychological stress was low. (2) The data sources were mainly from Guangdong (57.3\%), especially Guangzhou, which were related to the design and promotion of this online survey by a hospital in Guangzhou. A small number of participants from other regions, especially Hubei province $(3.1 \%)$, were involved in the investigation. (3) In terms of personnel composition, the medical personnel working at the front line represent only a tiny proportion $(10 \%)$ of the total. As to the front-line workers, the medical staff who work at the isolation wards exhibited significantly higher anxiety $(21.6 \%)$ or depression $(25.7 \%)$ screening positive rates in this present study. This finding was in line with a study focused on 332 front-line health professionals in Wuhan City, which reported the prevalence of anxiety and depression symptoms of $24.7 \%$ and $20.2 \% .^{11}$ In agreement with certain other studies, the nurse was also more vulnerable to anxiety or depression than doctors. ${ }^{11}{ }^{30}$ Furthermore, this study revealed another critical risk factor to the emotional disturbance that had not been emphasised much. Medical staff with one or more family members infected by COVID-19 appeared to be three to four times riskier to anxiety or depression than those not in such a situation. Thus, these medical staff should be more focused on psychological intervention. Allowing them to have adequate time to look after their families and relieving them of the guilt of causing the infection among their relatives may help in the psychological intervention. In China, by nationally activating medical staff from other provinces to Hubei and locally activating medical staff in other cities or hospitals to COVID-19-focused hospitals, the government could achieve enough medical staff at the front line and optimal work routine and rest time for medical workers. Certainly, the government nationally gathered enough personal prevention instruments for the health workers. All these measures from the national or the individual level have produced prevailing strength against the virus, and at the same time protect the body and mind health of the front-line medical staff.

Here are some limitations of this study. Due to suffering from somatic illness, restrictive treatment circumstances and the limitation of the casting range, it was difficult to acquire the patients' mental health data by either interviewing or online survey at the initial stage of the pandemic. The number of patients participating in this survey was comparably quite small compared with those of the public or medical staff groups. This sample difference perhaps influenced the reliability of the statistical results. The participants could not be instructed and supervised to complete the questionnaires independently, which may have caused several counts of missing values and the relatively low rate of responses for the ZSDS and ZSAS. All these might affect the statistical results. Scales of ZSAS and ZSDS could mainly evaluate the emotional symptoms and not directly reveal the stress reactions. Moreover, no other valid pathway was available to authenticate the respondents' identifications except by way of the entrances they chose.

\section{CONCLUSION}

In conclusion, the COVID-19 outbreak has resulted in varying extents of psychological effects on the public, medical staff and patients. Nearly half of the patients had anxiety and depression symptoms, according to this survey. The front-line medical staff, especially nurses and those with an infected family member, are at high risk of emotional disturbances. Regarding the public, youngsters, females, those with lower educational levels and people lacking masks are more likely to feel anxious or depressed. These populations require immediate psychological intervention in case of severe mental disorders. It is important to encourage the high-risk public or patients and the medical staff to seek psychiatric help from specialists and undergo clinical diagnosis and therapy by the psychiatrist. At the same time, the professionals should give more advice via various media for them to adjust to mind rebalance. The government should support more psychological intervention facilities for them to use, for example, make policy to build or strengthen online consulting, and more importantly, the online hospitals. People under great stress could easily get psychological help, even medicine therapy from specialists at the remote terminals. 
Contributors NG made the idea. XYW, JZ and NG designed the study and monitored the process. XLW adapted the primary data. XLW and JT completed the statistics. XLW, QZ, XHW, TL and WY wrote the initial manuscript. QZ, CZ and $\mathrm{XHW}$ were in charge of the language edition of the manuscript and completed the submission.

Funding The authors have not declared a specific grant for this research from any funding agency in the public, commercial or not-for-profit sectors.

Competing interests None declared.

Patient consent for publication Not required.

Ethics approval The ethical committee of the Third Affiliated Hospital of Sun Yatsen University approved the study ((2020) 02-023-01).

Provenance and peer review Not commissioned; externally peer reviewed.

Data availability statement Data are available upon reasonable request. The data sets used and/or analysed during the current study are available from the corresponding author on reasonable request.

Open access This is an open access article distributed in accordance with the Creative Commons Attribution Non Commercial (CC BY-NC 4.0) license, which permits others to distribute, remix, adapt, build upon this work non-commercially, and license their derivative works on different terms, provided the original work is properly cited, appropriate credit is given, any changes made indicated, and the use is non-commercial. See: http://creativecommons.org/licenses/by-nc/4.0/.

\section{ORCID iD}

Nianhong Guan http://orcid.org/0000-0001-8524-3586

\section{REFERENCES}

1 WHO. Coronavirus disease (COVID-19) dashboard. secondary coronavirus disease (COVID-19) dashboard, 2020. Available: https:// www.who.int/emergencies/diseases/novel-coronavirus-2019

2 Rogers JP, Chesney E, Oliver D, et al. Psychiatric and neuropsychiatric presentations associated with severe coronavirus infections: a systematic review and meta-analysis with comparison to the COVID-19 pandemic. Lancet Psychiatry 2020;7:611-27.

3 Troyer EA, Kohn JN, Hong S. Are we facing a crashing wave of neuropsychiatric sequelae of COVID-19? neuropsychiatric symptoms and potential immunologic mechanisms. Brain Behav Immun 2020;87:34-9.

4 da Silva FCT, Neto MLR. Psychiatric symptomatology associated with depression, anxiety, distress, and insomnia in health professionals working in patients affected by COVID-19: a systematic review with meta-analysis. Prog Neuropsychopharmacol Biol Psychiatry 2021;104:110057.

5 Qin X, Shu K, Wang M, et al. Mental health status of patients with coronavirus disease 2019 in Changsha. Zhong Nan Da Xue Xue Bao Yi Xue Ban 2020;45:657-64.

6 Salari N, Hosseinian-Far A, Jalali R, et al. Prevalence of stress, anxiety, depression among the general population during the COVID-19 pandemic: a systematic review and meta-analysis. Global Health 2020;16:57.

7 Ren X, Huang W, Pan H, et al. Mental health during the Covid-19 outbreak in China: a meta-analysis. Psychiatr Q 2020;91:1033-45.

8 Luo M, Guo L, Yu M, et al. The psychological and mental impact of coronavirus disease 2019 (COVID-19) on medical staff and general public - A systematic review and meta-analysis. Psychiatry Res 2020;291:113190.

9 Kassaw C, Pandey D. The current mental health crisis of COVID-19 pandemic among communities living in Gedeo zone Dilla, SNNP, Ethiopia, April 2020. J Psychosoc Rehabil Ment Health 2020:1-5.
10 Xiaoming X, Ming A, Su H, et al. The psychological status of 8817 Hospital workers during COVID-19 epidemic: a cross-sectional study in Chongqing. J Affect Disord 2020;276:555-61.

11 Wang $\mathrm{Y}$, Duan Z, Peng K, et al. Acute stress disorder among frontline health professionals during the COVID-19 outbreak: a structural equation modeling investigation. Psychosom Med 2021;83:373-9.

12 Preti E, Di Mattei V, Perego G, et al. The psychological impact of epidemic and pandemic outbreaks on healthcare workers: rapid review of the evidence. Curr Psychiatry Rep 2020;22:43.

13 Shu L. [Self-rating depression scale (SDS)]. In: Wang X, Wang X, $\mathrm{Ma} \mathrm{H}$, eds. Rating scales for mental health. Beijing: Chinese Mental Health Journal Publisher, 1999: 194-6.

14 Wu W. [Self-rating anxiety scale (SAS)]. In: Wang X, Wang X, Ma H, eds. Rating scales for mental health. Beijing: Chinese Mental Health Journal Publisher, 1999: 235-8.

15 Tang D, Pan C, Qi H. [The report on 70 cases of adolescents with depressive disorders]. Chin Mental Health J 2003;7:468-70.

16 Gong J, Chan RCK. Early maladaptive schemas as mediators between childhood maltreatment and later psychological distress among Chinese college students. Psychiatry Res 2018;259:493-500.

17 Zhang Y, Liu R, Li G, et al. The reliability and validity of a Chineseversion short health anxiety inventory: an investigation of university students. Neuropsychiatr Dis Treat 2015;11:1739-47.

18 Liu XC, Ma DD, Kurita H, et al. Self-Reported depressive symptoms among Chinese adolescents. Soc Psychiatry Psychiatr Epidemiol 1999;34:44-7.

19 Dunstan DA, Scott N. Assigning clinical significance and symptom severity using the Zung scales: levels of misclassification arising from confusion between index and RAW scores. Depress Res Treat 2018;2018:1-13

20 Zhang J, Tao J, Li X. Characteristics of psychiatric contact consultation in inpatients with the COVID-19 in Wuhan: a retrospective analysis. Chin J Psychiatry 2020;53:282-6.

21 Mak IWC, Chu CM, Pan PC, et al. Long-term psychiatric morbidities among SARS survivors. Gen Hosp Psychiatry 2009;31:318-26.

22 Huang C, Huang L, Wang Y, et al. 6-Month consequences of COVID-19 in patients discharged from Hospital: a cohort study. Lancet 2021;397:220-32.

$23 \mathrm{Wu}$ M, Han H, Lin T, et al. Prevalence and risk factors of mental distress in China during the outbreak of COVID-19: a national crosssectional survey. Brain Behav 2020;10:e01818.

24 Kassaw $C$. The magnitude of psychological problem and associated factor in response to COVID-19 pandemic among communities living in Addis Ababa, Ethiopia, March 2020: a cross-sectional study design. Psychol Res Behav Manag 2020;13:631-40.

25 Huang J, Liu F, Teng Z, et al. Public behavior change, perceptions, depression, and anxiety in relation to the COVID-19 outbreak. Open Forum Infect Dis 2020;7: ofaa273.

26 Cai Z, Zheng S, Huang Y, et al. Emotional and cognitive responses and behavioral coping of Chinese medical workers and general population during the pandemic of COVID-19. Int J Environ Res Public Health 2020;17:6198.

27 Ding Y, Xu J, Huang S, et al. Risk perception and depression in public health crises: evidence from the COVID-19 crisis in China. Int $J$ Environ Res Public Health 2020;17:17.

28 Qiu D, Li Y, Li L, et al. Policies to improve the mental health of people influenced by COVID-19 in China: a scoping review. Front Psychiatry 2020;11:588137.

29 Wang L-Q, Zhang M, Liu G-M, et al. Psychological impact of coronavirus disease (2019) (COVID-19) epidemic on medical staff in different posts in China: a multicenter study. J Psychiatr Res 2020;129:198-205.

30 Hacimusalar Y, Kahve AC, Yasar AB, et al. Anxiety and hopelessness levels in COVID-19 pandemic: a comparative study of healthcare professionals and other community sample in turkey. $J$ Psychiatr Res 2020;129:181-8. 